\title{
INTERPRETIVE SUMMARIES, AUGUST 2012
}

Granulomorphometry: A suitable tool for identifying hydrophobic and disulfide bonds in $\beta$-lactoglobulin aggregates. Application to the study of $\beta$-lactoglobulin aggregation mechanism between 70 and $95^{\circ} \mathrm{C}$. By Petit et al., page 4188 . $\beta$-Lactoglobulin is the predominant whey protein in dairy products and its denaturation behavior plays a major role in aggregation and fouling mechanisms of milk derivatives. Understanding how heating processes and physicochemical parameters influence $\beta$-lactoglobulin denaturation and the nature of aggregates binding interactions (covalent or hydrophobic) is a current challenge in the dairy field. This study presents the suitability of granulomorphometry for identifying the binding interactions involved in $\beta$-lactoglobulin aggregates. This new analytical method was validated and then applied to study the temperature influence on the aggregation mechanisms occurring in a $\beta$-lactoglobulin concentrate with moderate calcium content between 70 and $95^{\circ} \mathrm{C}$.

Consumer acceptance and sensory evaluation of Monti Dauni Meridionali Caciocavallo cheese. By Santillo et al., page 4203. The research was undertaken to study the sensorial attributes of Monti Dauni Meridionali Caciocavallo cheese produced according to 6 different production protocols. The results may help dairy operators to improve specific phases of Caciocavallo cheese production, which play a crucial role in defining cheese maturing and sensorial characteristics. Flavor, appearance, and texture attributes were correlated with proteolytic and lipolytic cheese indices. Consumer preference is oriented toward cheeses with an overall higher degree of maturity.

Incorporation of Lactobacillus casei in Iranian ultrafiltered Feta cheese made by partial replacement of $\mathrm{NaCl}$ with $\mathrm{KCl}$. By Karimi et al., page 4209. In this work, probiotic Iranian ultrafiltered Feta cheese was produced using Lactobacillus casei (as probiotic culture), adjunct thermophilic, and mesophilic lactic acid cultures, and potassium chloride (as partially replaced salt). The simultaneous effects of $L b$. casei and $\mathrm{KCl}$ on chemical, microbiological (viability of probiotic), physicochemical, and sensory properties of samples were investigated. This product is regarded a functional dairy product because it is probiotic with a low sodium level and has the potential to be commercially produced and widely consumed.

Effect of parity, days in milk, and milk yield on detailed milk protein composition in Mediterranean water buffalo. By Bonfatti et al., page 4223. Buffalo milk protein composition was quantified in a large sample of animals and the effects of nongenetic factors and milk yield on variation of buffalo milk protein fractions were estimated. Effects of polymorphisms at milk protein genes on contents and relative proportions of protein fractions should be investigated to get a better insight into variation of buffalo milk protein composition. Knowledge about factors affecting variation in protein composition is still scarce, and further research is needed so that concentrations of individual proteins could be altered to meet specific requirements.

Preparation and characterization of $\beta$-lactoglobulin hydrolysate-iron complexes. By Zhou et al., page 4230. Iron deficiency is prevalent in populations that consume mainly plant-based foods. It can cause several organ dysfunctions and lead to iron deficiency anemia. $\beta$-Lactoglobulin hydrolysate can bind with iron and form complexes, which may have potential as an effective delivery vehicle to maintain iron status.

Aggregation and conformational changes of bovine $\beta$-lactoglobulin subjected to dynamic high-pressure microfluidization in relation to antigenicity. By Zhong et al., page 423\%. Our previous research indicated that dynamic high-pressure microfluidization (DHPM) had a significant effect on the antigenicity of $\beta$-lactoglobulin $(\beta-\mathrm{LG})$. The present study was undertaken to unravel further the reason for changes in $\beta$-LG antigenicity. The aim of this work was to evaluate the aggregation and conformational changes of $\beta$-LG subjected to DHPM and to relate these changes to the antigenicity of $\beta-\mathrm{LG}$.

Active compounds and distinctive sensory features provided by American ginseng (Panax quinquefolius L.) extract in a new functional milk beverage. By Tárrega et al., page 4246. Ginsenosides are believed to be the active compounds in ginseng that enhance cognitive functions, but they are also responsible for the bitter taste in ginseng-containing products. For the first time, American ginseng has been added to functional milk as an ingredient. Ginsenosides were quantified after ultra-high-temperature (UHT) treatment, and the sensory profile was assessed. Ginsenosides are relatively stable to heat. The effects of ginsenosides and UHT treatment on the sensory profile of milk are discussed. The feasibility of adding ginsenosides in UHT-treated milk is an important implication for industries whose challenge is to produce functional foods with cognition-promoting properties.

Chemical, microbiological, textural, color, and sensory characteristics of pressed ewe milk 
cheeses with saffron (Crocus sativus L.) during ripening. By Licón et al., page 4263. Adding saffron to dairy products represents an innovative practice. Few studies have evaluated the influence of this spice on general aspects and ripening parameters of pressed ewe milk cheese. The main changes were observed on sensory characteristics and color. This work reveals the need for further studies regarding saffron antimicrobial activity and its properties as a flavor enhancer.

Short communication: Evaluation of bulk tank milk microbiological quality of nine dairy farms in Tennessee. By Gillespie et al., page 4275. Bulk tank milk (BTM) quality may be determined by various parameters including somatic cell count, standard plate count (SPC), preliminary incubation count (PIC), laboratory pasteurization count, and counts of Staphylococcus spp., Streptococcus spp., and coliforms. The purpose of this study was to evaluate the BTM quality of 9 Tennessee dairy farms, and to determine the relationship with selected quality milk parameters. Strong correlations were associated between PIC and SPC. Seasonal variations were noted for some milk quality parameters. Analysis of BTM gives us a snapshot of milk quality and provides producers with valuable information concerning the status of health and sanitation in their herds.

Short communication: Production of antihypertensive peptide HLPLP by enzymatic hydrolysis: Optimization by response surface methodology. By Quirós et al., page 4280. A new strategy for the production of antihypertensive peptide HLPLP by enzymatic hydrolysis is shown. Corolase PP was an appropriate enzyme to liberate this peptide from sodium caseinate. The obtained hydrolysate might be used as a functional ingredient with antihypertensive properties in the food industry.

Short-term increases in stocking density affect the lying and social behavior but not the productivity of lactating Holstein dairy cows. By Krawczel et al., page 4298. The effect of stocking density [100 (1 cow per resting and feeding space), 113, 131, and $142 \%$ ] on Holstein dairy cows over 2 wk was evaluated in this study. Lying time was reduced at 131 and $142 \%$, relative to 100 or $113 \%$. The percentage of time cows spent ruminating within freestalls decreased at 131 and $142 \%$, relative to $100 \%$. Displacements from the feed bunk increased as stocking density increased. Despite these differences, hygiene, fecal cortisol metabolites (assessment of a stress response), and productivity were not altered. Increased stocking density affected some aspects of behavior, but the resulting consequences are unclear.
Validation of the Finnish national dairy disease register-Data transfer from cow health cards to the disease register. By Rintakoski et al., page 4309. National dairy disease registers store information that is valuable for research and economic decision making. The accuracy of the Finnish dairy disease register was validated and reasons for data loss and register bias, such as underreporting, were recorded. Use of dairy registers in research is common but validation is rarely reported. Results of this study help to secure better estimates for disease incidence and can influence studies on animal welfare, epidemiology, and farm economy.

Antimicrobial resistance profiles of common mastitis pathogens on Canadian dairy farms. By Saini et al., page 4319. Monitoring of antimicrobial resistance in bacteria has clinical and public health significance. The present study determined antimicrobial resistance profiles of common bovine mastitis pathogens, including the prevalence of methicillin-resistant Staphylococcus aureus (MRSA), and extended-spectrum $\beta$-lactamase (ESBL) producing Escherichia coli and Klebsiella species isolates on 89 Canadian dairy farms. Antimicrobial susceptibility testing was done using Sensititre (Trek Diagnostic Systems Inc., Cleveland, $\mathrm{OH}$ ). Resistance to $\beta$-lactams (penicillin, ceftiofur, and cephalothin) was uncommon in Staph. aureus isolates. One MRSA isolate was confirmed. Most coliforms were resistant to tetracycline, whereas resistance to ceftriaxone and ciprofloxacin was absent. Multidrug resistance was commonly observed in coliforms. No ESBL producers were observed.

Herd-level risk factors associated with cow mortality in Swedish dairy herds. By Alvåsen et al., page 4352. Increasing dairy cow mortality (euthanasia and death) is an animal welfare problem that causes economic loss to the farmer and leads to a misuse of resources. This study demonstrated increasing mortality rates in Swedish dairy herds between 2002 and 2010. Larger herd size, longer calving intervals, and the Swedish Holstein breed were associated with greater mortality. Lower mortality was observed in herds with a higher herd-average milk yield, during autumn and winter and in organically managed herds. This information on herd-level risk factors is needed to reverse the increasing trend in on-farm cow mortality.

Evaluation of two doses of ceftiofur crystalline free acid sterile suspension for treatment of metritis in lactating dairy cows. By McLaughlin et al., page 4363. Cows with metritis within $10 \mathrm{~d}$ of calving were treated with saline (control) or ceftiofur crystalline free acid sterile suspension (CCFA-SS) twice, $72 \mathrm{~h}$ apart. The CCFA-SS treatment increased metritis clinical cure rate at $14 \mathrm{~d}$ and decreased rectal temperature 
during the first 5 or $6 \mathrm{~d}$ after treatment. Treatments were administered safely in commercial dairy facilities, and injection sites were normal in at least $98.6 \%$ of cows 9 wk later. Thus, CCFA-SS administered twice, 72 $\mathrm{h}$ apart, to cows with metritis was safe and significantly increased the metritis clinical cure rate.

Combined use of Ovsynch and a progesterone supplementation after artificial insemination in dairy cattle. By Forro et al., page 4372. An early and well-established progesterone synthesis at the beginning of pregnancy has the most advantageous effect on the development of the embryo. Synchronization of ovulation has become very popular in modern dairy farms, but can lead to decreased pregnancies per artificial insemination $(\mathrm{P} / \mathrm{AI})$. Progesterone supplementation postinsemination can be a useful tool to improve $\mathrm{P} /$ AI of cows synchronized with different protocols. The better-conditioned cows showed a greater improvement in $\mathrm{P} / \mathrm{AI}$ after the progesterone supplementation compared with cows having low body condition score.

Prevalence, antimicrobial resistance, and molecular characterization of methicillin-resistant Staphylococcus aureus from bulk tank milk of dairy herds. By Kreausukon et al., page 4382. Methicillin-resistant Staphylococcus aureus (MRSA) has been identified as an emerging pathogen in livestock animals that is readily transferable to humans being in contact with livestock. The objective of the study was to estimate the prevalence of MRSA in bulk tank milk from German dairy herds and to characterize the isolates. Livestock-associated MRSA of clonal complex CC398 does occur in German dairy herds. They belong to 2 different spa types, are resistant to a number of antimicrobials used in livestock farming, and do not carry major virulence-associated genes. Isolates of Staph. aureus should always be tested for resistance to oxacillin or cefoxitin for early detection of MRSA occurrence in herds.

Characteristics and retention of luteal structures, extended post-insemination cycle, progesterone, and pregnancy-specific protein B in serum after human chorionic gonadotropin treatment of dairy cows. By Stevenson and Pulley, page 4396. Treatment of dairy cows $7 \mathrm{~d}$ after artificial insemination (AI) with human chorionic gonadotropin (hCG) compared with saline induced new luteal structures in $70 \%$ of cows, regardless of pregnancy or pretreatment luteal status, and increased progesterone concentrations, as expected. Pregnancy per AI at first service, however, was not increased by hCG, particularly in hCG cows that had 1 pretreatment corpus luteum (CL) and did not respond to hCG. Regardless of treatment, $25 \%$ of cows that retained an original
CL to d 28 were not pregnant at $\mathrm{d} 32$. Retention of original CL indicated that pregnancy was initiated but failed, as verified by concentrations of progesterone and pregnancy-specific protein B.

Microscopic differential cell counting to identify inflammatory reactions in dairy cow quarter milk samples. By Pilla et al., page 4410. The diagnosis of mastitis is based on somatic cell counting and bacteriological analysis; differential cell counting (DCC) identifies early changes in the relative cell populations. The aim of the study was to identify cytological parameters to classify mammary quarters as healthy or diseased in the field. Single percentages of lymphocytes, polymorphonuclear neutrophilic leukocytes (PMNL), or macrophages were evaluated first, and then combinations of cell populations were tested to increase the discrimination power of DCC. The highest Fisher value was that of $\log$ PMNL:lymphocyte ratio. Microscopic DCC has potential to be a useful tool to identify cows affected by any inflammatory process of the mammary gland.

The effects of overstocking Holstein dairy cattle during the dry period on cortisol secretion and energy metabolism. By Huzzey et al., page 4421. Four groups of 10 nonlactating, late-gestation Holstein dairy cattle were exposed to two 14-d stocking density treatments using a replicated crossover design: (1) control (1 lying stall/cow and $0.67 \mathrm{~m}$ of feed bunk space/cow) and (2) overstocked (1 lying stall $/ 2$ cows and $0.34 \mathrm{~m}$ of linear feed bunk space/cow). The energy metabolism of cattle was altered during the overstocked treatment, as evidenced by impaired glucose clearance and an attenuated insulin response during a glucose tolerance test. Daily nonesterified fatty acid concentrations were also higher during overstocking despite greater average daily intakes. Fecal cortisol metabolite concentrations tended to be greater during overstocking. Overstocking dairy cattle can result in physiological changes.

Short communication: Relationship between competitive success during displacements at an overstocked feed bunk and measures of physiology and behavior in Holstein dairy cattle. By Huzzey et al., page 4434. The objective of this study was to evaluate how a cow's level of success during competitive interactions at an overstocked feed bunk is related to its behavior and physiological health. Cows that were displaced more frequently than they could displace others (low-success cows) showed evidence of insulin resistance; they also had greater plasma free fatty acid and fecal cortisol metabolite concentrations relative to cows with high competitive success. Feeding behavior was unaffected by competition success. 
Low-success cows have a physiological profile that may increase their risk for future health complications.

Short communication: Molecular typing of Prototheca zopfii from bovine mastitis in Japan. By Sobukawa et al., page 4442. Prototheca zopfii is an achlorophyllic alga that causes bovine mastitis, resulting in a reduction in milk production and the secretion of thin watery milk with white flakes. In this study, the $18 \mathrm{~S}$ ribosomal DNA sequences of diverse clinical specimens from different areas in Japan were studied to clarify the pathogenicity of P. zopfii var. zopfii genotypes. All isolates from bovine mastitis in Japan were identified as P. zopfii genotype 2. Therefore, bovine mastitis caused by $P$. zopfii would mainly be transmitted from cows infected with $P$. zopfii var. zopfii genotype 2 .

Short communication: Evaluation of vaginal discharge following treatment with a progesterone insert. By Fischer-Tenhagen et al., page 444\%. Purulent vaginal discharge is often seen in cows treated with an intravaginal hormone insert to improve fertility. This study evaluated the origin and importance of this discharge. Samples collected from the vagina and uteri of dairy heifers treated with an intravaginal insert were examined bacteriologically and cytologically. An infection of vagina and uterus with common pyogenic pathogens was found after treatment.

Technical note: Data loggers are a valid method for assessing the feeding behavior of dairy cows using the Calan Broadbent Feeding System. By Krawczel et al., page 4452. This study evaluated the agreement between 2 methods (continuous review of video data and a change-of-state data logger) for determining the feeding time and feed bin visits of dairy cows. The agreement of the methods was established from the lack of mean or slope biases and containment of more than $95 \%$ of the data within the maximum allowable difference $( \pm 20$ min of feeding time and \pm 3 visits to the feed bin). This indicates the data loggers were a valid method for measuring the feeding behavior, which eliminates the labor-intensive review of video data.

Milk production, nitrogen balance, and fiber digestibility prediction of corn, whole-plant grain sorghum, and forage sorghum silages in the dairy cow. By Colombini et al., page $445 \%$. Sorghum is a low-input forage crop that can be used in replacement of corn silage under certain circumstances (water shortage and corn parasites). Three diets based alternatively on corn, whole-plant grain sorghum, or forage sorghum silages were tested on lactating cows. Forage sorghum silage determined a lower milk yield because of the lower dry matter intake, which might be attributable to the excessive length of chopping of the silage. Milk urea and urinary nitrogen excretion were higher for forage sorghum diet. Fiber digestibility predicted by a feeding model was lower than in vivo values.

Effect of supplementary concentrate type on nitrogen partitioning in early lactation dairy cows offered perennial ryegrass-based pasture. By Whelan et al., page 4468. Dietary manipulation to reduce nitrogen excretion is environmentally advantageous. In this experiment, reducing concentrate crude protein, supplementing low crude protein concentrates with a methionine source, and replacing rolled barley with ground corn in low-crude protein concentrates were evaluated for effects on milk production and nitrogen partitioning in early lactation dairy cows offered grazed pasture. Reducing concentrate crude protein reduced milk yield, nitrogen intake, and excretion in the urine and milk. However, offering supplementary methionine or replacing rolled barley with ground corn maintained milk yield and milk nitrogen while reducing nitrogen excretion in the urine.

Effects of starch content of calf starter on growth and rumen $\mathrm{pH}$ in Holstein calves during the weaning transition. By Laarman et al., page 4478. To facilitate rumen development, calves are typically fed readily fermentable calf starters with a high dietary starch concentration, providing the calf rumen with sufficient volatile fatty acids, while at the same time risking ruminal acidosis. We found that decreasing dietary starch concentration in calf starter did not increase rumen $\mathrm{pH}$, but that consumption of calf starter with faster digestion rates was accompanied by more severe ruminal acidosis without affecting growth rates.

Protein fractionation byproduct from canola meal for dairy cattle. By Heendeniya et al., page 4488. This study aimed to systematically evaluate fiberprotein as feed ingredient in ruminant diets in terms of chemical profiles, energy value, rumen-degradation characteristics, intestinal digestion, and modeling nutrient supply of the fiber-protein fraction as dietary component for dairy cattle in comparison with commercial canola meal and soybean meal.

Novel use of the wild species Cephalaria joppensis for silage preparation and its nutritive value for feeding lactating dairy cows. By Miron et al., page 4501. The wild plant species Cephalaria joppensis (CJ) was domesticated to produce forage for ruminants. The CJ tended to have higher crop mass yield per hectare than wheat and similar digestibility. Freshly harvested CJ used as is or CJ-based silage can be packed in polyethylene bales to produce ensiled total mixed rations. Total mixed rations containing CJ silages are characterized by a long outdoor shelf-life of 6 mo, high stability under aerobic exposure, and nutritive 
value for sheep and dairy cows similar to that of wheat silage.

Development of colonic microflora as assessed by pyrosequencing in dairy calves fed waste milk. By Edrington et al., page 4519. The objective of the current study was to examine the effect of pasteurization of waste milk used to feed dairy calves on the bacterial diversity of their lower gut. Fecal samples from dairy calves ( 1 wk to 6 mo old) fed pasteurized or nonpasteurized waste milk were analyzed for bacterial diversity. In general, bacterial diversity was greater for the calves fed pasteurized waste milk at all ages (except 1 wk of age) and increased with age in both treatments. Salmonella, when detected, was most often in the younger animals fed nonpasteurized milk.

Effects of feeding dairy cows different legumegrass silages on milk phytoestrogen concentration. By Höjer et al., page 4526. Phytoestrogens are hormone-like substances in plants, some of which influence human health (positively or negatively), notably providing protection against certain cancers and that are transferred to the milk when fed to dairy cows. In this study, milk concentrations of isoflavone phytoestrogens were higher, and those of lignans lower, when cows were fed red clover silage than when fed white clover- or birdsfoot trefoil-grass silage. Milk concentrations of equol, an isoflavone metabolite, were high, but varied substantially among cows, despite similar intake.

Effect of supplementary concentrate type on milk production and metabolic status in early lactation dairy cows grazing perennial ryegrass-based pasture. By Whelan et al., page 4541. Supplementing grazing dairy cows is often important to maximize production and prevent excessive body tissue mobilization. The type of concentrate used may have an important influence on both outcomes. In this experiment, reducing concentrate crude protein $(\mathrm{CP})$ concentration, offering supplementary methionine, and replacing rolled barley with ground corn in low-CP concentrates were evaluated for effects on milk production and metabolic status. Reducing concentrate $\mathrm{CP}$ reduced milk and protein yields. However, offering supplementary methionine or substituting rolled barley with ground corn ameliorated these effects without negatively affecting the metabolic status of the early lactation dairy cow.

Production response to corn silage produced from normal, brown midrib, or waxy corn hybrids. By Barlow et al., page 4550. Numerous corn hybrid varieties are available for producing silage, including many specialty hybrids such as brown midrib (BMR) and waxy. The BMR hybrids have lower lignin concentrations, which have been shown to support higher intake and milk yield. The starch in waxy corn hybrids is $100 \%$ amlyopectin, which is more digestible than that of normal corn hybrids, but data are limited on its use for silage. This trial was conducted to determine the production response of lactating dairy cows to diets based on silage produced from normal, BMR, or waxy hybrids. The BMR hybrids supported greater milk yield, whereas milk protein was highest with the waxy hybrid and milk fat was lowest for normal hybrids.

Factors associated with ruminal $\mathrm{pH}$ at the herd level. By Geishauser et al., page 4556. Subacute ruminal acidosis may harm health and lower productivity of dairy cows. Rumen acidosis is usually diagnosed by measuring ruminal fluid $\mathrm{pH}$. Ruminal fluid $\mathrm{pH}$ has been studied in individual cows, whereas studies at the herd level are scarce. Little information is available on when to expect low ruminal $\mathrm{pH}$ along lactation. This paper describes rumen fluid $\mathrm{pH}$ dynamics at the herd level. Four hundred thirty-two cows of a commercial dairy herd were rumen sampled, volume and $\mathrm{pH}$ of the samples were measured, and effects of age, ration composition, stage of lactation, time of day, and milk yield were evaluated. The percentage of concentrates in the ration, days in milk, time of day, and daily milk yield were significant factors affecting ruminal $\mathrm{pH}$ at the herd level.

Immune responses in lactating Holstein cows supplemented with $\mathrm{Cu}, \mathrm{Mn}$, and $\mathrm{Zn}$ as sulfates or methionine hydroxy analogue chelates. By Nemec et al., page 4568. Lactating dairy cows were fed for 12 wk rations containing $\mathrm{Cu}, \mathrm{Mn}$, and $\mathrm{Zn}$ supplemented at $100 \%$ of requirements as inorganic sulfates or chelates. Compared to the sulfate minerals, cows fed the chelates had increased antibody titer response to a model vaccination. Subsets of cows fed the chelated minerals also had increased milk $\mathrm{Cu}$ and increased neutrophil phagocytosis. Supplementation with more available forms of $\mathrm{Cu}, \mathrm{Mn}$, and $\mathrm{Zn}$ may enhance immune response of dairy cows.

Supplementation of increasing amounts of linseed oil to dairy cows fed total mixed rations: Effect on digestion, ruminal fermentation characteristics, protozoal populations, and milk fatty acid composition. By Benchaar et al., page 4578. This study was conducted to evaluate the effects of including increasing amounts $(2,3$, or $4 \%$ of dietary dry matter) of linseed oil in total mixed rations (50:50 forage:concentrate ratio) on digestion, ruminal fermentation, milk production, and milk fatty acid composition. Adding linseed oil up to $4 \%$ of dietary dry matter enhanced the milk fat content of potential health beneficial fatty acids (n-3 fatty acids) without negatively affecting rumen function, digestion, and hence, cow performance. 
Short communication: Evaluation of serum immunoglobulin G concentrations using an automated turbidimetric immunoassay in dairy calves. By Alley et al., page 4596. Failure of passive transfer is commonly recognized as a significant risk factor for morbidity and mortality in dairy calves. Although multiple methods are available to assess adequacy of passive transfer, none of these are ideal. The objective of this study was to evaluate a commercially available immunoassay that can rapidly measure immunoglobulin $\mathrm{G}$ ( $\mathrm{IgG}$ ) concentrations in calves using a portable analyzer. The assay performed very well compared with radial immunodiffusion and could be used by dairy producers or veterinarians (or both) to monitor IgG concentrations on farms.

Hot topic: Effect of breeding strategies using genomic information on fitness and health. $B y$ Egger-Danner et al., page 4600. Different total merit indices (with and without the inclusion of direct health traits and increased weights for fertility and udder health indexes) and breeding strategies [a conventional progeny-testing program and genomically enhanced breeding programs $(50 \%$ and $100 \%$ of inseminations by young bulls)] were analyzed. The relative annual monetary genetic gain for fitness traits is increased by genomic selection programs. Including direct health traits changes the annual genetic response for fertility and udder health in a positive direction. The direction of the genetic trend is determined by specific weights in the total merit index. When a clear positive trend is observed, genomically enhanced breeding programs can reinforce it.

Association of DGAT1 genotype, fatty acid composition, and concentration of copper in milk with spontaneous oxidized flavor. By Juhlin et al., page 4610. Milk concentrations of copper and unsaturated fatty acids showed strong and unfavorable associations with development of spontaneous oxidative flavor in milk. Moreover, the K232A allele of the diacylglycerol acyltransferase 1 (DGAT1) gene was associated with a markedly higher off-flavor risk. To maintain the good taste of milk, consideration of these factors might be necessary in future breeding decisions.

Joint estimation of genetic parameters for testday somatic cell count and mastitis in the United Kingdom. By Mrode et al., page 4618. Mastitis, a costly disease for dairy farms worldwide, affects production, welfare, and milk quality. Direct and indirect selection via somatic cell count is expected to reduce mastitis incidence. Until recently, mastitis records have been insufficient in number and quality to be included in genetic evaluations in the UK. In the present study, genetic parameters for test-day somatic cell count and mastitis were estimated using multitrait random regres- sion models. The proposed model is expected to result in more accurate genetic evaluations for udder health compared with the currently used repeatability model for lactation-average somatic cell count.

Computational strategies for national integration of phenotypic, genomic, and pedigree data in a single-step best linear unbiased prediction. By Legarra and Ducrocq, page 4629. Genomic evaluations can include pedigree, genotypes, and records through the so-called single-step method. Its large-scale implementation may seem difficult, given the complexity of operations involved. We show that this complexity can be avoided by choosing an iterative procedure (national pedigree evaluation + genomic evaluation) or by including additional unknowns. Such approaches allow genetic evaluations including records, pedigree, and genotypes to become routine.

Long-term selection strategies for complex traits using high-density genetic markers. By Kemper et al., page 4646. Most breeding programs in dairy cattle select animals with the highest overall genetic merit. This paper compares this approach to a more strategic method aiming to realize a predefined ultimate genotype. We conclude that the selection of animals with the highest overall merit is most appropriate for dairy cattle, given their low reproductive rate, low tolerance of inbreeding, and the highly polygenic nature of traits important for dairying.

Comparison of genomic predictions using medium-density $(\sim 54,000)$ and high-density $(\sim 777,000)$ single nucleotide polymorphism markers in Nordic Holstein and Red dairy cattle populations. By Su et al., page 465\%. Accuracies of genomic predictions are expected to be improved by increasing the density of single nucleotide polymorphism (SNP) markers. This study investigated genomic predictions in Nordic Holstein and Red dairy cattle (RDC) using 54,000- (54K) and imputed 777,000$(777 \mathrm{~K})$ marker data. On average, the $777 \mathrm{~K}$ markers led to an increase in prediction reliability by 0.5 percentage points in Holsteins, and 1.0 in RDC, and a reduction of bias, compared with the $54 \mathrm{~K}$ markers. Overall, the extra gain from $777 \mathrm{~K}$ markers was small. Further studies are needed to exploit the potential advantage of high-density markers in genomic prediction.

Temperature and relative humidity influence the microbial and physicochemical characteristics of Camembert-type cheese ripening. $B y$ Leclercq-Perlat et al., page 4666. We evaluated the effects of temperature and relative humidity $(\mathrm{RH})$ on the ripening kinetics of Camembert, microbial growth, and biochemical evolutions under different temperatures and relative humidities. Some factors depended solely 
on temperature, such as yeast growth and the $\mathrm{pH}$ in the rind. However, our findings highlighted the fact that the interactions between temperature and $\mathrm{RH}$ play a role in the case of Penicillium camemberti sporulation, Brevibacterium aurantiacum growth, carbon substrate consumption rates, and the thickening of the cheese underrind. The best ripening conditions to achieve optimum between-microorganism growth and biochemical kinetics were $13^{\circ} \mathrm{C}$ and $94 \% \mathrm{RH}$.

A simple formulation and solution to the replacement problem: A practical tool to assess the economic cow value, the value of a new pregnancy, and the cost of a pregnancy loss. By Cabrera, page 4683. Assessing the cow value and making replacement decisions in dairy farming have important economic implications. A simple calculation of the replacement problem was developed with the aim to better assist dairy farmers in their continuous decisions of keeping or replacing cows. This simple calculation achieved the same trend decisions previously found with more complex models and was better suited to create a user-friendly and practical decision support system that farmers could use to make more effective and efficient replacement decisions. The tool integrates farmer's intuition and knowledge within a dynamic and interactive process for better decisions.

Retrospective evaluation of health event data recording on 50 dairies using Dairy Comp 305. By Wenz and Giebel, page 4699. Current dairy health records are user defined and are highly variable within and between farms. They typically lack the accuracy and consistency needed for herd-level summary and evaluation to better inform management decisions.

How do dairy cows chew? Particle size analysis of selected feeds with different particle length distributions and of respective ingested bolus particles. By Schadt et al., page 470\%. Not only feed but also respective bolus particle size might alter diet efficiency and cow performance. Measurements of feed and bolus particle sizes showed that rye grass hays were chewed to constant sizes, independent of their original sizes, as long as hay particles were long enough to be retained on the $8-\mathrm{mm}$ screen of the Penn State Particle Separator. Particles from a total mixed ration could be swallowed apparently longer compared with rye grass hay. Bolus particle size might be related to feed chemical composition but is not necessarily associated to feed particle size.

International genetic evaluation of Holstein bulls for overall type traits and body condition score. By Battagin et al., page 4721. This study documented differences in trait definitions and use of breeding values among countries that participate in the International Bull Evaluation for body condition score, overall conformation, udder, and feet and leg scores of Holstein bulls. Genetic correlations were used to identify distances among countries. Trait definitions of the overall traits were used to explain the distances. Large variation exists among countries in definition of overall conformation traits and trait harmonization among countries may improve the genetic correlations. 